\title{
Tabularia
}

\section{La solennisation des fêtes dans la vie liturgique et temporelle des moines du Mont Saint-Michel (XIII ${ }^{\mathrm{e}}$ $\mathrm{XV}^{\mathrm{e}}$ siècles)}

The Solemnization of Feasts in Liturgical and Temporal Life of the Monks of Mont Saint-Michel (13th-15th Centuries)

La solennità delle feste nella vita liturgica e temporale dei monaci del Mont

Saint-Michel (XIII-XV secolo)

Feierliches Erinnern im liturgischen und weltlichen Leben der Mönche von Mont Saint-Michel (13.-15. Jahrhundert)

\section{Louis Chevalier}

\section{OpenEdition}

Journals

Édition électronique

URL : https://journals.openedition.org/tabularia/5036

DOI : $10.4000 /$ tabularia.5036

ISSN : $1630-7364$

Éditeur :

CRAHAM - Centre Michel de Boüard, Presses universitaires de Caen

\section{Référence électronique}

Louis Chevalier "La solennisation des fêtes dans la vie liturgique et temporelle des moines du Mont Saint-Michel (XIIIe-XVe siècles) », Tabularia [En ligne], Autour de la Bibliothèque virtuelle du Mont SaintMichel, mis en ligne le 25 mai 2021, consulté le 27 mai 2021. URL : http://journals.openedition.org/ tabularia/5036 ; DOI : https://doi.org/10.4000/tabularia.5036

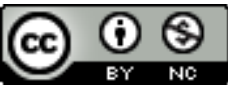

la revue Tabularia est mise à disposition selon les termes de la Licence Creative Commons Attribution - Pas d'Utilisation Commerciale 4.0 International. 

La solennisation des fêtes

dans la vie liturgique et temporelle des moines du Mont Saint-Michel (XIII ${ }^{\mathrm{e}}-\mathrm{XV}^{\mathrm{e}}$ siècles)

The Solemnization of Feasts

in Liturgical and Temporal Life of the Monks

of Mont Saint-Michel (13th-15th Centuries)

La solennità delle feste

nella vita liturgica e temporale dei monaci

del Mont Saint-Michel (XIII-XV secolo)

Feierliches Erinnern

im liturgischen und weltlichen Leben der Mönche

von Mont Saint-Michel (13.-15. Jahrhundert)

Louis CHEVALIER

Docteur - CRAHAM (UMR 6273)

Université de Caen Normandie/CNRS

louis.chevalier@hotmail.fr

Résumé:

L'analyse des calendriers liturgiques, des ordinaires et du cérémonial du Mont SaintMichel du point de vue des rits éclaire l'histoire du culte des saints et de la vie liturgique et temporelle de l'abbaye. Le degré de solennité distingue, dans les manuscrits, les fêtes selon leur importance, par l'indication du nombre de leçons à l'office de matines et de cierges allumés dans le chœur de l'église, et par la mention d'ornements liturgiques. Cet article examine, à travers une étude codicologique et textuelle de livres liturgiques en usage entre le XIII ${ }^{e}$ et le $\mathrm{XV}^{\mathrm{e}}$ siècle au Mont Saint-Michel, la catégorisation hiérarchique des fêtes montoises et son évolution à la fin du Moyen Âge, ainsi que les conséquences de la solennisation des fêtes dans la vie temporelle des moines.

Mots-clés: Mont Saint-Michel, liturgie, calendrier liturgique, Ordinaire, degrés de solennité

Abstract:

Analysis of the liturgical calendars, the ordinals and the ceremonial of Mont Saint-Michel, and particularly the degrees of solemnity, can inform us about the history of the cult of the saints and the liturgical and temporal life of the abbey. The degrees of solemnity, as markers of solemnization in the manuscripts, differentiate feasts according to their importance, indicating the number of lessons at the hour of matins and the candles lit in the choir, and mentioning

Tabularia "Études», "Autour de la Bibliothèque virtuelle...", 2021, p. 1-18, 25 mai 2021 URL: http://journals.openedition.org/tabularia/5036 | DOI: 10.4000/tabularia.5036 
the liturgical ornaments. Through a codicological and textual study of liturgical books in use between the thirteenth and fifteenth centuries at Mont Saint-Michel, this article examines the hierarchical categorisation of the feasts in the liturgy and the evolution of the degrees of solemnity on Mont Saint-Michel at the end of the Middle Ages, and the significance of the solemnization of a feast for the temporal life of the monks.

Keywords: Mont Saint-Michel, Liturgy, liturgical calendar, Ordinal, degrees of solemnity

Riassunto:

L'analisi dei calendari liturgici, degli ordinari e del cerimoniale di Mont Saint-Michel da un punto di vista rituale consente di ricostruire la storia del culto dei santi, della liturgia $e$ della vita dei monaci all'interno dell'abbazia. I dettagli rituali registrati nei manoscritti (numero delle lezioni mattutine e dei ceri accesi nel coro della chiesa, menzione di ornamenti liturgici) sono marchi di solennità che conferiscono alle feste un diverso grado di importanza. Attraverso uno studio codicologico e testuale dei libri liturgici in uso tra il XIII e il XV secolo a Mont Saint-Michel, questo articolo esamina la caratterizzazione gerarchica delle feste dell'abbazia micaelica e la sua evoluzione alla fine del Medioevo, nonché le conseguenze della solennizzazione delle feste nella vita quotidiana dei monaci.

Parole chiave: Mont Saint-Michel, liturgia, Calendario liturgico, Ordinario, marchi di solennità

Abstrakt:

Die Analyse der liturgischen Kalender, der Ordinarien und Zeremonialbücher vom Mont Saint-Michel unter dem Blickpunkt der feierlichen Gedächtnistage verdeutlicht die Geschichte der Heiligenkulte und des liturgischen und täglichen Lebens in der Abtei. Die Ordnung der Gedächtnistage in den Manuskripten unterscheidet die Feiertage nach ihrer Bedeutung, indem die Anzahl der Lesungen im morgendlichen Gottesdienst und die der entzündeten Kerzen im Chorraum der Kirche festgelegt werden sowie die liturgischen Ornamente bestimmt werden. Dieser Beitrag untersucht mit Hilfe einer kodikologischen und Textuntersuchung der liturgischen Bücher, die zwischen dem 13. und 15. Jahrhundert in Mont Saint-Michel verwendet wurden, die hierarchische Ordnung der mönchischen Feiern und ihre Entwicklung am Ende des Mittelalters, sowie die Auswirkungen des feierlichen Erinnerns auf das tägliche Leben der Mönche.

Schlagworte: Mont Saint-Michel, Liturgie, liturgischer Kalender, Ordinarien, Gewichtung der Feiertage

Notes de l'auteur - Les recherches présentées dans cet article ont été réalisées dans le cadre d'une thèse soutenue en 2019: je remercie mes directrices de thèse Véronique Gazeau et Catherine Jacquemard de leurs conseils précieux.

Le fonds des manuscrits liturgiques copiés pour l'abbaye du Mont SaintMichel est aujourd'hui composé de trois livres de codification liturgique (un cérémonial et deux ordinaires), de quatre livres de la messe (un sacramentaire conservé en deux parties, un missel et un évangéliaire-nocturnal également utilisé pour la célébration de l'office), de onze livres de l'office (trois bréviaires, un collectaire, cinq lectionnaires, un légendier et un martyrologe) et d'un 
pontifical, produits entre le $\mathrm{XI}^{\mathrm{e}}$ et le $\mathrm{XV}^{\mathrm{e}}$ siècle ${ }^{1}$. L'usage liturgique du Mont Saint-Michel peut être identifié dans ces manuscrits grâce à l'étude du cursus et du cérémonial qu'ils décrivent, et par l'examen de leur sanctoral et du degré de solennité attribué dans leur calendrier aux fêtes de l'année liturgique. Je souhaiterais analyser ici le degré de solennité des fêtes du sanctoral montois et son évolution entre le XIII et le XV ${ }^{e}$ siècle, dans la perspective de l'histoire du culte des saints et d'une étude de la vie temporelle des moines du Mont SaintMichel, en m'appuyant sur la critique des calendriers, des ordinaires et du cérémonial qui recèlent de précieuses indications sur le rang hiérarchique des fêtes et sur l'ordonnance de la liturgie ${ }^{2}$. L'ordinaire codifie les rites, l'itinéraire des processions ou encore le choix des pièces des féries et des fêtes de l'année liturgique, et parfois certaines pratiques non cultuelles comme la consommation de boissons durant les processions: il indique également les règles de résolution des cas d'occurrence de fêtes du temporal et du sanctoral ${ }^{3}$. Le cérémonial donne des règles générales pour la célébration du culte - énonçant par exemple le rôle des acteurs de la liturgie - sans évoquer les particularités de chaque fête ${ }^{4}$. Le cérémonial et l'ordinaire, qui ne sont pas des livres de chœur, ne contiennent pas le texte entier des lectures, des chants et des oraisons mais seulement des incipit.

1. Bréviaires: Avranches, Bibl. mun., ms 39 (XIII s.), bréviaire d'hiver; Paris, BnF, ms nouv. acq. lat. $424\left(\mathrm{XV}^{\mathrm{e}} \mathrm{s}\right.$.), bréviaire d'été; Maredsous, Bibliothèque de l'abbaye, $\mathrm{ms} 16^{\circ} / 1\left(\mathrm{XV}^{\mathrm{e}} \mathrm{s}\right.$.). Cérémonial: Avranches, Bibl. mun., ms 214 (XVe s.). Collectaire: Avranches, Bibl. mun., ms 215 (XV s.). Évangéliaire-nocturnal: Avranches, Bibl. mun., ms 44 (XIII s.). Lectionnaires: Avranches, Bibl. mun., ms 128 (XIe-XII ${ }^{e}$ s.); Avranches, Bibl. mun., ms 129 (XIe s.); Avranches, Bibl. mun., ms 131 (XIII s.) ; Avranches, Bibl. mun., ms 168, fol. 1r-92r (XIII-XVe s.); Avranches, Bibl. mun., ms 214 (XVe s.). Martyrologe: Avranches, Bibl. mun., ms 214, (XVe s.). Missel: Avranches, Bibl. mun., ms 42 (XIII s.). Légendier: Avranches, Bibl. mun., ms 167 (XIII s.). Nécrologes: Avranches, Bibl. mun., ms 214 (XVe s.); Avranches, Bibl. mun., ms 215 (XVe s.). Ordinaires: Avranches, Bibl. mun., ms 46 (XIV s.); Avranches, Bibl. mun., ms 216 (XV s.). Pontifical: Paris, BnF, ms lat. 14832 (XII ${ }^{e}$ s.). Sacramentaire: New York, Pierpont Morgan Library, ms 641 (XIe s.-XII ${ }^{e}$ s.); Rouen, Bibliothèque patrimoniale Villon, ms suppl. 116 (mm 15) $\left(\mathrm{XI}^{e} \mathrm{~s}\right.$.-XII ${ }^{e}$ s.). Ces fragments de sacramentaire sont deux parties d'un même manuscrit selon Henri Tardif (cf. TARDIF, 1967, p. 358). L'origine montoise de fragments d'évangéliaires conservés à Avranches et Saint-Pétersbourg est discutée (Avranches, Bibl. mun., ms 48, fol. 1r-3v; Avranches, Bibl. mun., ms 66, fol. 1r-1v; Avranches, Bibl. mun., ms 71, fol. 1r-1v. Saint-Pétersbourg, Bibliothèque nationale de Russie, ms lat. O.V.I).

2. Calendriers liturgiques du Mont Saint-Michel: Avranches, Bibl. mun., ms 39 (fol. 29v-32r); Avranches, Bibl. mun., ms 42 (fol. 2v-8r); Avranches, Bibl. mun., ms 214 (p. I-XIII); Avranches, Bibl. mun., ms 215 (1r-6v); Avranches, Bibl. mun., ms 216 (fol. 4v-10v); Paris, BnF, ms nouv. acq. lat. 424 (fol. $7 \mathrm{r}-12 \mathrm{r}$ ); Maredsous, Bibliothèque de l'abbaye, ms 16\% (fol. 1r-6v).

3. Cf. Cоllomb, 1995, p. 97-109. Et: Martimort, 1991, p. 62-67. L'Ordinaire 46 fut vraisemblablement copié entre 1330 et 1370 , et l'Ordinaire 216 entre 1386 et 1432 . Si le texte des deux manuscrits est très proche, leurs prescriptions ne sont cependant pas toujours identiques: l'ordo décrit par l'Ordinaire 46 est sans doute antérieur à celui présenté dans l'Ordinaire 216. Les moines semblent avoir continué à corriger l'Ordinaire 46 après la copie de l'Ordinaire 216, au moins jusque dans la première moitié du XVe siècle. Cf. Chevalier, 2019, vol. 1, p. 72-80 et p. 90-98.

4. Cf. Martimort, 1991, p. 89-9o. Joseph Lemarié date le cérémonial du Mont Saint-Michel (Avranches, Bibl. mun., ms 214) du début du XVe siècle: Lemarié, 1967, p. 307. 
Après avoir présenté les marqueurs de solennité utilisés dans les manuscrits puis la hiérarchie des fêtes en vigueur au Mont Saint-Michel, j'étudierai la relation existant entre degrés de solennité et menu des repas du convent, afin d'éclairer le phénomène de solennisation de la vie monastique en ses différentes réalités lors des fêtes majeures.

\section{Les marqueurs de solennité}

Dans les calendriers et les livres liturgiques médiévaux, le degré de solennisation d'une fête est exprimé par différents marqueurs. Les rits sont, parmi ceux-ci, les plus aisément repérables. Jean-Baptiste Lebigue définit ces courtes mentions comme des «critères renvoyant à des pratiques cultuelles ou cantorales distinctives, et dont l'expression, nécessairement succincte, est apposée à l'intitulé de la fête » ${ }^{5}$. Les manuscrits du Mont Saint-Michel présentent des rits de cinq types: en premier lieu, la mention: «In capis» fait référence aux chapes portées par les officiants au cours des vêpres et de la messe. En deuxième lieu, la mention: «In albis» désigne des aubes. En troisième lieu, les mentions: «Tres lectiones» et: «Duodecim lectiones» indiquent le nombre de leçons des nocturnes, qui peut varier de trois (office de type férial) à douze (office de type dominical) ${ }^{6}$. Les mentions: «Quatuor lectiones», "Quinque lectiones» ou "Octo lectiones», qui suivent l'intitulé de certaines fêtes, ne participent pas de l'expression des degrés de solennité, signifiant que seules quatre, cinq ou huit leçons de l'office de matines sont choisies, lors d'une journée liturgique, en relation avec la fête du jour ou avec une fête célébrée quelques jours plus tôt qui possède une octave. En quatrième lieu, les mentions: "Tribus cereis», "Quinque cereis», "Septem cereis», "Nouem cereis» et "Triginta cereis» correspondent au nombre de cierges allumés au-dessus du chœur, conformément à l'expression plusieurs fois répétée dans les ordinaires: «Super chorum». L'utilisation de trente cierges semble une particularité liturgique du Mont Saint-Michel: cette pratique est associée dans les ordinaires aux fêtes majeures de l'abbaye, telles que la fête de saint Aubert, fondateur de l'abbaye, ou les fêtes de l'Épiphanie, de l'Annonciation, ou de l'Apparition de saint Michel sur le Mont Gargan. En cinquième lieu, la mention: «Memoria» (ou «Commemoratio») fait état dans la plupart des cas d'une fête du plus bas échelon - et dans quelques autres cas d'une fête inférieure à une autre fête fixée le même jour. Ces différents critères, indiquant le degré de solennité des fêtes, permettaient aux moines de résoudre les cas de fêtes occurrentes: dans ce système universel, une fête de rit In capis est supérieure à une fête de

5. Lebigue, 2012, p. 355

6. Ibid., p. 355-356: [les offices de type dominical,] «plus solennels, comptent trois nocturnes à matines et deux offices de vêpres, comme un dimanche, au lieu d'un seul nocturne et une seule heure de vêpres pour le type férial, c'est-à-dire le modèle suivi pour les jours de la semaine (féries)». 
rit In albis, qui est elle-même supérieure à une fête de rit Duodecim lectiones. Outre les mentions de rits, l'attribution d'une vigile (jour précédant la fête) et d'une octave à une fête souligne également son importance ${ }^{7}$.

À titre d'exemple, nous relevons dans le calendrier du Bréviaire $16^{\circ} 1$ ( $\mathrm{XV}^{e}$ siècle), au mois de décembre, les rits XII lectiones, in capis attribués à la fête de saint Nicolas (6 décembre) et à la fête de la Conception de la Vierge (8 décembre), les rits XII lectiones, in albis attribués à la fête de sainte Lucie (13 décembre) et la mention Commemoratio accompagnant la fête de saint Damase (11 décembre) ${ }^{8}$ (voir fig. 1 ci-après).

\section{Degrés de solennité et usages liturgiques}

Le degré de solennité attribué à une fête suggérait directement aux moines le cérémonial à déployer au long de la journée: le Cérémonial 214, qui différencie les fêtes à douze leçons et les fêtes à trois leçons, lie ainsi à chacune de ces deux classes des rites précis ${ }^{9}$. Dans son importante étude consacrée aux ordinaires et au cérémonial du Mont Saint-Michel, Joseph Lemarié a mis en évidence, en partant d'une classification à six échelons des degrés de solennité, les variations du cérémonial montois selon le rattachement d'une fête à l'une de ces classes - montrant ainsi que lors des grandes solennités (Pâques, Nativité, Pentecôte, Assomption, fête de saint Michel du 29 septembre, fête de la Dédicace du Mont Saint-Michel [16 octobre], Toussaint), fêtes In capis et à trente cierges, les autels de l'abbatiale étaient aspergés d'eau bénite et encensés par le sacristain accompagné d'autres officiers de la liturgie, durant l'office de matines et après l'office de complies, au chant d'une antienne et d'une oraison.

Dom Lemarié montra également qu'aux fêtes à trois leçons, un diacre, un sous-diacre en aubes, et deux acolytes et un thuriféraire officiaient auprès du prêtre aux deux messes du jour ${ }^{10}$. Jean-Baptiste Lebigue a conclu à la synchronie, dans le dernier tiers du XII e siècle, de l'introduction des rits dans les manuscrits et de la rédaction des premiers ordinaires, livres de codification liturgique qui fondent la hiérarchie des fêtes ${ }^{11}$.

7. L'Ordinaire 216 décrit la vigile des fêtes de Jean-Baptiste (fol. 130v), Pierre et Paul (fol. 133v), Jacques le Majeur (fol. 141v), Laurent (fol. 146v), Matthieu (fol. 162), Michel (28 septembre, fol. 164), Simon et Jude (fol. 17ov) et de la Toussaint (fol. 171v). Cet ordinaire livre en outre la liturgie des octaves des fêtes d'Étienne (fol. 39v), Jean (fol. 4ov), des Saints-Innocents (fol. 41), Aubert (fol. 132), Jean-Baptiste (fol. 135v), Pierre et Paul (fol. 137), Benoît (fol. 139), Laurent (fol. 15ov), de l'Assomption de la Vierge (fol. 152v), de la Nativité de la Vierge (fol. 161), Michel (6 octobre, fol. 167), de la Dédicace (fol. 170) et de Martin (18 novembre, fol. 178).

8. Maredsous, Bibliothèque de l'abbaye, $\mathrm{ms} 16 / 1^{\circ}$, fol. $6 \mathrm{v}$.

9. Avranches, Bibl. mun., ms 214, p. 209-212 et p. 215-221.

10. LemariÉ, 1967 , p. 324-326.

11. Lebigue, 2014, p. 41-42. 


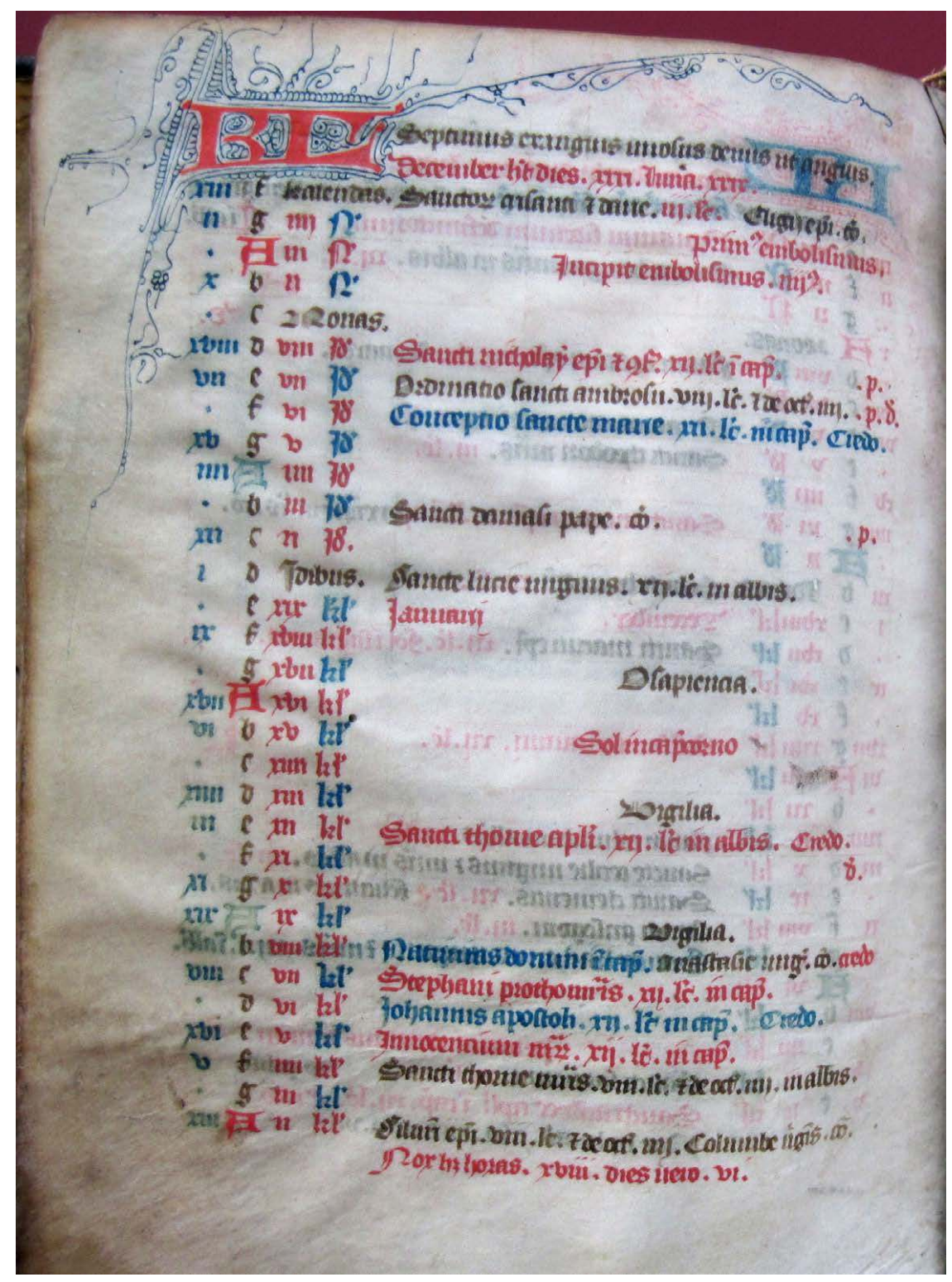

Fig. 1 - Maredsous, Bibliothèque de l'abbaye, $\mathrm{ms} \mathrm{16 \% 1,} \mathrm{fol.} 6 \mathrm{v}$. Avec l'aimable autorisation de la Bibliothèque 
Nous pouvons déduire d'un classement des rits utilisés dans les manuscrits montois une hiérarchie cohérente des fêtes du Mont Saint-Michel selon leur degré de solennité. Dans l'étude précitée, Joseph Lemarié établit une classification des fêtes comprenant les degrés de solennité suivants: grandes solennités, solennités In capis cum XXX cereis, solennités In capis cum VII cereis, fêtes à douze leçons In albis cum V cereis, fêtes à douze leçons Cum tribus cereis et fêtes à trois leçons ${ }^{12}$. En partant de cette classification et en y ajoutant quelques échelons, nous pouvons ordonner les fêtes du sanctoral montois selon la catégorisation hiérarchique suivante, à l'étude des ordinaires:1) Fêtes in capis et à douze leçons (à trente cierges, neuf cierges ou sept cierges). 2) Fêtes in albis, à douze leçons et cinq cierges. 3) Fêtes à douze leçons et trois cierges. 4) Fêtes à trois leçons. 5) Memoria.

Le résultat de cette catégorisation des fêtes, qui correspond à un état liturgique datant de 1330-1430, est présenté en annexe. Les fêtes du plus haut degré (fêtes in capis) sont dans le sanctoral les fêtes de la Vierge (Conception, Nativité, Annonciation, Purification et Assomption de la Vierge), les fêtes de Jean-Baptiste et des Apôtres, les fêtes de l'archange Michel (fête du 29 septembre, et fête de l'Apparition de Michel sur le Mont Gargan), la fête d'Aubert, fondateur du sanctuaire, et la fête de la Dédicace du sanctuaire, et les fêtes de Benoît, Catherine, Martin et Laurent. Le degré de solennité des fêtes semble équivalent dans les deux ordinaires: les différences de rits qui séparent ces manuscrits résultent probablement de simples omissions du copiste ou d'une normalisation des usages. En effet, les fêtes qui dans le premier ordinaire étaient de rits In albis et Duodecim lectiones et ne comportaient pas de mentions de cierges ont toutes reçu dans le deuxième ordinaire le rit Quinque cereis, et les fêtes de rit Duodecim lectiones qui n'étaient pas accompagnées de mentions de cierges dans le premier ordinaire ont reçu le rit Tribus cereis dans le deuxième ordinaire.

Parmi les fêtes normandes citées dans le sanctoral de l'Ordinaire 216, nous comptons une fête de rit Memoria (fête de Berthevin de Parigny, martyr au $\mathrm{X}^{\mathrm{e}}$ siècle dans la Manche), une fête à trois cierges (fête de Philibert, abbé de Jumièges au VII e siècle), une fête à trois leçons (fête de Leufroy, abbé de Sainte-Croix dans le diocèse d'Évreux au VIII ${ }^{e}$ siècle), une fête à douze leçons et trois cierges (fête de Paterne, évêque d'Avranches au VI ${ }^{\mathrm{e}}$ siècle), une fête In albis à cinq cierges (fête de Wandrille, abbé de Fontenelle au VII e siècle), une fête In albis, à douze leçons et cinq cierges (fête de saint Ouen, archevêque de Rouen au VII siècle) et deux fêtes en chapes, à douze leçons et trente cierges (fête d'Aubert, évêque d'Avranches au VIII ${ }^{e}$ siècle, et fête de la Dédicace du Mont Saint-Michel).

Les fêtes furent copiées de couleur rouge ou de couleur noire dans les calendriers dichromes de l'Ordinaire 216 et du Collectaire 215. Certaines fêtes sont également écrites de couleur bleue dans les calendriers du Missel 42, du Cérémonial 214 et du Bréviaire $16 \%$. Ce code de couleurs était utilisé au Moyen Âge pour distinguer d'un seul regard les solennités des fêtes de

12. LEMARIÉ, 1967 , p. 324-326. 
moindre importance, suivant un usage qui se répandit à partir du XIII ${ }^{e}$ siècle dans les établissements religieux ${ }^{13}$. Cependant, ce système de hiérarchisation par le choix de couleurs ne fut pas toujours appliqué avec une rigueur égale dans les manuscrits, le travail du copiste pouvant également obéir à un souci esthétique ${ }^{14}$. L'observation matérielle des calendriers comparée à la hiérarchie des fêtes reconstituée plus tôt montre que le calendrier du Bréviaire $16 \% 1$ est le seul témoin montois à présenter un usage parfaitement cohérent de la couleur en conformité avec l'indication des rits: la couleur bleue distingue dans ce témoin les grandes solennités (fêtes de la Nativité, de saint Aubert, de la Dédicace, de la Toussaint...), les fêtes de moindre importance y étant copiées en rouge, ou pour le plus petit échelon en noir ${ }^{15}$.

Le degré de solennité des fêtes put évoluer au cours du temps, conduisant à la correction des rits au sein des manuscrits. L'étude génétique de l'Ordinaire 46 révèle ainsi les variations qui traversèrent, au cours des $\mathrm{XIV}^{\mathrm{e}}$ et $\mathrm{XV}^{\mathrm{e}}$ siècles, la vie liturgique du Mont Saint-Michel: ont été dans ses pages corrigés de seconde main les rits des fêtes de la Circoncision du Seigneur (p. 22), de l'Épiphanie (p. 27), de Lucie (fol. 87v), Benoît (fol. 97r), de l'Annonciation (fol. 97v), de Marc (fol. 99v), Jean (fol. 102v), Barnabé (fol. 106v), Jean-Baptiste (fol. 11or) et Marie-Madeleine (fol. 119r). Dans le cas de la fête de Jean-Baptiste, le rit Triginta cereis a par exemple été ajouté par grattage et réécriture au rit Duodecim lectiones, dans le but de rehausser la solennité de cette fête. Joseph Lemarié a mis en exergue dix-huit fêtes dont la solennité a été relevée après le XIV ${ }^{\mathrm{e}}$ siècle dans la liturgie montoise, citant les fêtes de Catherine, Marie-Madeleine, Mathias, Benoît, Marc, Jean, Barnabé, Jérôme, Cécile, Clément, Lucie, Grégoire, les fêtes de la Chaire de Pierre, de Nicolas et de sa Translation, des Saints-Innocents et les deux fêtes $\mathrm{d}^{\mathrm{A}}$ Ambroise ${ }^{16}$. Nous pouvons ajouter à cette liste la fête d'Anne: cette fête est de rit Tres lectiones dans l'Ordinaire 216 (fol. $8 \mathrm{r}$ et fol. 181v), le Collectaire 215 (fol. $4 \mathrm{r}$ ) et le Bréviaire nouv. acq. lat. 424 (fol. 1or) mais de rits In albis et Duodecim lectiones dans le Cérémonial 214 (p. VII) et le Bréviaire $16 \% 1$ (fol. $4 \mathrm{r}$ ). Le degré de solennité de la fête d'Anne fut rehaussé à la fin du Moyen Âge: inscrite de seconde main

13. Lebigue, 2014, p. 60 .

14. Ibid., p. 62-63: "À partir du milieu du XIII ${ }^{\mathrm{e}}$ siècle, le rôle de la polychromie est surtout de souligner la hiérarchie des rits. La rigueur avec laquelle ce principe est appliqué dépend du lieu et du destinataire du manuscrit».

15. Dans le calendrier du bréviaire conservé à Maredsous, on trouve copiées de couleur bleue les fêtes de la Conception de la Vierge, de la Nativité du Seigneur, de Jean, de la Circoncision du Seigneur, de l'Annonciation, de l'Épiphanie, de Jean-Baptiste, de l'Assomption, de la Nativité de la Vierge, de Michel du 29 septembre, de la Dédicace du Mont Saint-Michel, de la Toussaint et de Catherine. La couleur rouge met en valeur les fêtes d'André, Nicolas, Thomas apôtre, Étienne, des Saints-Innocents, de Sébastien, Fabien (fête sans doute présentée en rouge car prenant place le même jour et sur la même ligne que la fête de Sébastien), Vincent, de la Conversion de Paul, de la Purification de la Vierge, d'Agathe, de la Chaire de Pierre, de Mathias, Benoît, de Pâques, de Marc, Barnabé, Aubert, Pierre et Paul, de la Translation de Martin, de la Translation de Benoît, de Marie-Madeleine, Jacques apôtre, Pierre, de l'Inventio d'Étienne, de Laurent, Barthélemy, de la Décollation de Jean-Baptiste, de l'Exaltation de la sainte Croix, de Matthieu, Maurice, Denis, Luc, Simon et Jude, des fidèles défunts et de Martin.

16. LeMARIÉ, 1967, p. 346 
dans le corps de l'Ordinaire 216 (annoncée, fol. 142r, par la mention: «Quaere in fine» et présentée en fin de volume, fol. 181r) copié entre 1386 et 1432, et de première main dans son calendrier produit postérieurement à son texte liturgique en 1432, cette fête fut donc introduite dans la liturgie montoise au début du $\mathrm{XV}^{\mathrm{e}}$ siècle, et devint en peu de décennies une fête à douze leçons. La dévotion grandissante de la communauté envers Anne put naître, dans un contexte de dispute entre maculistes et immaculistes, de la vénération des moines pour la Vierge, dont ils célébraient les fêtes avec le plus haut degré de solennité. Les textes de l'office de sainte Anne présentés dans le Bréviaire $16 \% 1$ honorent en effet en Anne la génitrice de la Mère de Dieu ${ }^{17}$. Comme l'écrit Nicolas Trotin, «si l'on reconnaît à la Vierge Marie un caractère éminent de sainteté alors qu'apparait son rôle de médiatrice et d'avocate des hommes auprès du trône divin par la grâce de la prédestination qui l'a marquée de son sceau, alors ses parents ont été également sanctifiés $»^{18}$. Les moines du Mont Saint-Michel possédaient des reliques de sainte Anne depuis au moins 1396, date de rédaction d'un inventaire des reliques dans lequel sainte Anne est citée ${ }^{19}$.

Les rits résultaient de choix liturgiques, et leur évolution s'accompagnait d'un remaniement des usages. L'absence d'un ordinaire montois postérieur à l'Ordinaire 216 ne nous permet pas de mesurer le nombre de changements intervenus dans le cérémonial de cette fête après la modification de son degré de solennité, mais nous observons, grâce à la comparaison de l'Ordinaire 216 et du Bréviaire $16 \%$, un bouleversement du cursus canendi de son office (l'office du Bréviaire $16 \%$ étant plus fortement solennisé que l'office décrit dans l'Ordinaire 216, le cursus présenté dans le bréviaire doit être le plus tardif). $\mathrm{Au}$ commencement de l'office des matines, l'invitatoire Regi Sidereo remplace ainsi, dans le Bréviaire $16 \%$, l'invitatoire Regem omnium sanctorum donné par l'Ordinaire 216: seize pièces de chant (antiennes, répons, hymne et invitatoire) furent de cette manière remplacées aux offices des matines, des laudes, de prime et de tierce de cette fête. L'Ordinaire 216 et le Bréviaire $16 \% 1$ présentent deux états distincts de l'office d'Anne: l'étude génétique de l'Ordinaire 216 révèle par ailleurs deux états intermédiaires ayant existé avant la copie du bréviaire. En premier lieu, l'ordinaire a possédé un premier invitatoire dont

17. Citons par exemple l'oraison de l'office de none (variante de Corpus Orationum 1366 a): «Deus, qui beate Anne tantam gratiam donare dignatus es, ut beatissimam matrem tuam in suo gloriosissimo utero portare meruit, da nobis per intercessionem matris et filie tue propitiationis habundantiam, ut quarum commemorationem pro amore amplectimur, earum precibus ad celestem patriam pervenire valeamus» (Maredsous, Bibliothèque de l'abbaye, $\mathrm{ms} 16 \% 1$, fol. 450 ). Frappante également est l'hymne des laudes, dont la première strophe est: "Adesto nobis inclita, Dei matris O genitrix, [Anna,] que in celi perpetua cum Christo regnas gloria» (Maredsous, Bibliothèque de l'abbaye, $\mathrm{ms} 16 \%$, fol. 450). Le répertoire Analecta Hymnica signale la présence de cette hymne dans un bréviaire à l'usage de la collégiale du Saint-Sépulcre de Caen et dans des bréviaires de Coutances et de Sées, ainsi que dans un bréviaire franciscain, et dans des bréviaires à l'usage d'établissements d'Amiens: Dreves, 1895, p. 61-62.

18. Trotin, 2011, p. 156. Une étude comparative des manuscrits liturgiques normands pourrait permettre de déterminer s'il exista un modèle normand de l'office de sainte Anne.

19. Avranches, Bibl. mun., ms 213, fol. 158v. Cf. Dubois, 1967, p. 520 et p. 539. 
l'incipit, gratté, est aujourd'hui illisible, qui a été remplacé par la pièce: Regem omnium sanctorum. Le choix de l'invitatoire a de nouveau changé lorsqu'on a copié le Bréviaire $16 \%$. En second lieu, le manuscrit 216 eut originellement pour hymne des laudes une pièce qui a également été remplacée par grattage, cette fois par un chant que l'on retrouve dans le bréviaire: Adesto nobis inclita. Pour ce qui concerne la messe de cette fête, la comparaison de l'Ordinaire 216 avec une liste de pièces copiée de seconde main au $\mathrm{XV}^{\mathrm{e}}$ siècle dans le Missel 42 (fol. 8v) ne révèle aucune modification. La comparaison de l'office du Mont Saint-Michel, en ses différents états, avec l'office fécampois de sainte Anne ne fait pas apparaître de ressemblances significatives ${ }^{20}$, malgré la parenté de ces deux liturgies.

Le rehaussement du degré de solennité de plusieurs fêtes au cours des $\mathrm{XIV}^{\mathrm{e}}$ et $\mathrm{XV}^{\mathrm{e}}$ siècles témoigne, en conclusion, d'un phénomène de solennisation croissante de la liturgie du Mont Saint-Michel à la fin du Moyen Âge.

\section{Solennisation des fêtes et vie temporelle}

La succession des offices, la juxtaposition des calendriers naturel et liturgique, les lectures de la liturgie et du réfectoire tournaient constamment les moines vers l'Évangile et la commémoration des saints. À ce titre, nous pouvons nous demander quel écho la solennisation liturgique des fêtes trouvait dans la vie temporelle de la communauté. Les ordinaires du Mont Saint-Michel présentent le menu des repas de plusieurs fêtes du temporal et du sanctoral, nous donnant matière à comparaison entre cet aspect de la vie temporelle et les rits liturgiques.

Les Ordinaires 46 et 216 mentionnent, dans le corps de leur texte liturgique, à la suite de l'office de sexte ou de l'office de none, le menu des fêtes à douze leçons, qu'elles soient fêtes in albis ou in capis: l'Ordinaire 46 présente le menu de quarante-deux fêtes et l'Ordinaire 216 celui de quarante-quatre fêtes. Par leurs indications, les deux manuscrits ne décrivent que les plats qui étaient ajoutés en certaines occasions au repas quotidien, composé de deux plats cuits, d'une livre de pain et d'un plat de fruits ou de légumes crus $^{21}$. Les moines prenaient leurs repas dans un réfectoire accessible par le cloître, situé au plus haut de l'abbaye; la cuisine ouvrait de plain-pied sur le réfectoire. Le cellier se trouvait au premier niveau de l'abbaye.

Selon les prescriptions des ordinaires, le repas de la communauté était amélioré les jours de fête par l'ajout de certains légumes (fabae fractae: écrasée de fèves; album porretum: porée blanche ${ }^{22}$ ), de pâtisseries (gastellus: gâteau; flatones: flans), de vins (caritatem vini; pigmentum: vin miellé), ainsi que de mets divers (cena frixa: mets rôtis; sagimen: saindoux; ova: œufs; caseum: fromage;

20. L'office fécampois de sainte Anne, ajouté de seconde main dans la marge du folio 126v de l'ordinaire de l'abbaye (Fécamp, Musée du Palais Bénédictine, ms 186), a été édité par David Chadd dans: The Ordinal of the Abbey..., 1999-2002, p. 711-712: «The Feast of St Anne (Jul. 26)».

21. Cf. CABY, 2008, p. 289.

22. La porée blanche est une soupe, une sauce ou une purée composée de blancs de poireaux. 
butirum: beurre), d'une pitance (pitantia), supplément composé d'œufs et de fromage cuit ou cru, ou du général (generalis), autre supplément et portion individuelle contenant plusieurs œufs et du fromage cuit ${ }^{23}$. L'Ordinaire 46 présente treize menus distincts dont huit ne se rencontrent qu'à une seule fête. L'Ordinaire 216 inclut de la même manière dix-huit menus différents dont neuf sont attribués une seule fois.

Les ordinaires furent copiés ou utilisés au cours de la guerre de Cent Ans, alors que le Mont Saint-Michel subissait le siège de l'armée anglaise : l'abbaye ne recevait alors d'approvisionnement que par voie de terre à marée basse, et par voie maritime depuis la Bretagne de laquelle lui parvenaient froment et vin ${ }^{24}$. Les moines recueillaient leur eau à la fontaine Saint-Aubert, auprès de l'abbaye, ou dans une citerne construite entre 1417 et 1418 à la demande de l'abbé Robert Jolivet et citée dans un traité de 1420 établi entre les moines et le capitaine du Mont $^{25}$, ainsi que dans une fontaine construite dans les bâtiments de fortification entre 1417 et $1420^{26}$. D'après le traité de 1420 , les moines jouissaient en outre de jardins, d'un élevage de chevaux et de bœufs et des services d'un boucher ${ }^{27}$. La communauté profitait, d'après le traité, de l'aide d'un poissonnier et de poissons pêchés dans plusieurs cours d'eau de la région: saumons, esturgeons et marsouins ${ }^{28}$.

La mention de repas dans un livre liturgique s'explique sans doute à la fois par le besoin de régler, à destination des moines chargés de la cuisine, le menu des fêtes importantes, et par la conception bénédictine du repas, lieu de communion spirituelle des moines à l'instar exact, d'après saint Benoît, de l'office divin ${ }^{29}$ : c'est ce que révèlent en particulier les chapitres 24 et 25 de la règle qui évoquent l'excommunication mineure qui peut frapper un moine fautif. L'excommunication a mensa, premier degré de cette punition, conduit à une exclusion temporaire de la table commune, et à de menues interdictions dans la pratique liturgique. Le deuxième degré est l'exclusion à la fois de la table commune et du culte collectif, et l'interdiction faite à tout moine de bénir le repas du frère pris en faute. Le chapitre 35 de la règle établit par ailleurs

23. CABY, 2008, p. 289.

24. FIASSON, 2018, p. 220-221.

25. Cf. "Traité pour la défense du Mont Saint-Michel en 1440 », 1846, p. 212-213. Ce traité daterait plutôt de 1420: cf. FiAsson, 2014, p. 63, note 68.

26. FiASSON, 2018, p. 218-219.

27. "Traité pour la défense du Mont Saint-Michel en 1440 », 1846, p. 212.

28. Ibid., p. 213: "Que les ditz religieux aient la prise de poisson sur les marchants ou pescheurs venans a la dicte ville ainsi comme ils en ont joy tous les temps passés. Et aussi du droit qu'ils ont ès rivieres de Senne et de Coynon davoir les saulmons et aultres poissons. Ainsi Monseigneur le consent pourveu que en nourriture tout soit en commun. Que les ditz religieux aient les esturgeons et les marsouins prins ou trouves es greves et qu'ils en puissent joyr ainsi que leur appartient selon leurs tiltres et possessions. Monseigneur le consent comme dessus. Que ou cas que les dis marchans ou pescheurs ne feroient leur devoir d'apporter aux dits religieux les ditz esturgeons, marsouins saulmons et aultres poissons, toutes fois que les cas s'offriraient les dicts religieux les puissent et doivent punir par leur justice ainsi qu'il leur appartient et qu'ils en ont joy le temps passé. Monseigneur le consent».

29. Cf. Boulc'H, 1997, p. 315-317. 
un lien entre le service de la table et la prière liturgique, en demandant que les hebdomadiers chargés de servir le repas au réfectoire soient bénis dans l'oratoire le dimanche après l'office de matines en présence des autres frères de la communauté, ainsi que l'accomplissement d'un rite de lavement des pieds qui reproduit l'épisode de la Cène. Le chapitre 38 évoque la lecture qui accompagne le repas pris en silence et la bénédiction dans l'oratoire, par tous les frères, du lecteur de la semaine.

Les moines du Mont Saint-Michel eurent le souci de faire coïncider le plus justement possible composition des repas et rits liturgiques, en enrichissant les menus les jours de solennité en signe de fête. Un passage du Coutumier 214 livre ainsi une classification des repas en relation avec certains rits, indiquant par ailleurs le menu de plusieurs grandes fêtes:

A refectorio singularitas remoueatur, maxime in exquisitis cibariis et in uno uino tamen debiliori, si potatur, excepto. In festivitatibus XXX cereorum conuentus habeat duas pitantias et generale et cenam frixam et abbas uel magister ordinis ad scalam IX fercula, non ut sibi incorporent, sed ut aliis, prout expedire uiderint, largiantur. In festivitatibus IX cereorum, II pitancias et unum generale et ad scalam IX fercula. In festivitatibus VII cereorum, II pitencias et I generale et ad scalam IX fercula. In festivitatibus V cereorum, I pitanciam et I generale et ad scalam VII fercula. Qui ad scalam sedet, habeat ad minus VII fercula ad primum prandium et quinque tantummodo ad secundum. Die Pasche et tribus diebus sequentibus habeat conuentus flacones et gastellos; et tribus diebus ante Rogationes flacones; et die Ascensionis gastellos habeant et flacones; die Penthecostes et tribus diebus sequentibus et in festo beati Michaelis in maio et in omnibus festis in capis conuentus gastellos habeat et bonum uinum pro pigmento. In Quadragesima monachi panem breatum habeant et etiam simirellos. Die Jovis absoluto, uinum habeant et pigmentum et optimam pitanciam de coquina ${ }^{30}$.

Les rits ainsi pris en considération concernent les fêtes à trente, neuf, sept et cinq cierges, et les fêtes en chapes. Le repas des fêtes à trente cierges était composé de deux pitances, du général et de mets rôtis; le repas des fêtes à neuf cierges ou à sept cierges, de deux pitances et du général; les repas des fêtes à cinq cierges, d'une pitance et du général. Le jour de la fête et les trois premiers jours de l'octave de Pâques et le jour de l'Ascension, le convent recevait flans et gâteaux, et du flan à l'occasion des Rogations. Lors de la fête de Pentecôte et les trois jours suivants, et lors de la fête de l'Apparition de saint Michel sur le Mont Gargan et des différentes fêtes en chapes, le repas était agrémenté d'un vin miellé issu d'un

30. Avranches, Bibl. mun., ms 214, p. 6-7. La fin de ce texte est également présentée, avec de menues variantes, dans l'Ordinaire 216 (fol. 95r): «Die Pasche et tribus diebus sequentibus habeat conuentus flacones et gastellos; et tribus diebus ante Rogationes flatones; et die Ascensionis gastellos et flacones habeat; die Penthecostes et tribus diebus sequentibus et in festo beati Michaelis in maio pigmentum et in omnibus festis in capis conuentus gastellos habeat et bonum uinum pro pigmento. In Quadragesima monachi panem breatum habeant et etiam simirellos. Die Jouis absoluto, uinum habeant et pigmentum et optimam pitanciam de coquina». Plusieurs passages du coutumier ont été transcrits et commentés par Léon Guilloreau, in: GuilloreaU, 1915, p. 161-176. 
vin de qualité, et de gâteaux. Les moines recevaient du pain durant le Carême, période de jeûne ${ }^{31}$. Le Jeudi saint, du vin miellé et une excellente pitance leur étaient donnés.

Les menus décrits dans les ordinaires témoignent de la même réalité, comme le révèle la liste suivante d'attribution de pitances aux fêtes du temporal et du sanctoral établie à l'étude des prescriptions de l'Ordinaire $216^{32}$ :

- quatre pitances: fêtes de Michel du 29 septembre et de la Toussaint (quatre pitances, général, vin miellé, gâteaux). Fêtes de l'Annonciation, de l'Apparition de saint Michel sur le Mont Gargan et d'Aubert (quatre pitances, gâteaux, vin miellé). Fête de la Conception de la Vierge (quatre pitances, gâteaux, vin miellé). Dimanche de Pâques et dimanche de Pentecôte (quatre pitances, gâteaux, vin miellé, flans, mets rôtis). Fêtes de la Nativité du Seigneur et de l'Assomption de la Vierge (quatre pitances, gâteaux, vin miellé, mets rôtis);

- trois pitances: fêtes de Jean et de l'Épiphanie (trois pitances, gâteaux, mets rôtis). Fête de Laurent (trois pitances, gâteaux, vin miellé). Fête de Martin du 11 novembre (trois pitances, vin). Fêtes de la Purification et de la Nativité de la Vierge (trois pitances, général). Fêtes de Jean-Baptiste et de Catherine (trois pitances);

- deux pitances: fêtes d'André et de Nicolas (deux pitances, gâteaux). Fête de Pierre et Paul, et fête de la Translation de Benoît (deux pitances);

- une pitance: Samedi saint (une pitance, vin). Fête des Saints-Innocents (une pitance, mets rôtis). Rogations (une pitance, flans). Lundi de Pâques, mardi de Pâques, samedi de Pentecôte, premier dimanche après la Pentecôte, et fêtes de la Circoncision du Seigneur, de Thomas apôtre, Agnès, Vincent, de la Conversion de Paul, d'Agathe, Benoît (21 mars), Marc, Philippe et Jacques apôtres, de l'Inventio de la Sainte Croix, de Paul, de la Translation de Martin, de Marie-Madeleine, Wandrille, Jacques apôtre, Pierre, de l'Inventio d'Étienne, d'Agapit, de l'octave de l'Assomption, de Barthélemy, Ouen,

31. Cf. règle, Chapitre 49: «Ergo his diebus augeamus nobis aliquid solito pensu seruitutis nostrae, orationes peculiares, ciborum et potus abstinentiam ». La règle de saint Benoît interdit par ailleurs la consommation de viande de quadrupède durant toute l'année: «Carnium uero quadrupedum omnimodo ab omnibus abstineatur comestio, praeter omnino debiles aegrotos». Cette interdiction est reprise dans les Statuts de Grégoire IX copiés à la suite du coutumier dans le manuscrit Avranches, Bibl. mun., ms 214, p. 11: "In infirmitorio autem nullus carnes comedat, nisi monachus uel conuersus qui infirmus fuerit, aut propter debilitatem corporis ad infirmitorium missus».

32. Les prescriptions des deux ordinaires sont en cette matière presque identiques. Seulement, lors de la fête des Saints-Innocents l'Ordinaire 46 prescrit une pitance, une écrasée de fèves, de la porée blanche et des mets rôtis (fol. 18r) et l'Ordinaire 216 une pitance et des mets rôtis (fol. $36 \mathrm{v}$ ); et en la fête de saint Jean, l'Ordinaire 46 commande trois pitances, des gâteaux, une écrasée de fèves, de la porée blanche et des mets rôtis (fol. 17r) et l'Ordinaire 216 trois pitances, des gâteaux et des mets rôtis (fol. $35 \mathrm{v}$ ), conséquemment dans les deux cas à une probable omission du copiste de l'Ordinaire 216. Le cas de la solennité de saint Jean-Baptiste est différent: le menu de cette fête comprend deux pitances dans l'Ordinaire 46 (fol. 111r) et trois pitances dans l'Ordinaire 216 (fol. 132r), signe d'un enrichissement de ce repas. 
Augustin, de la Décollation de Jean-Baptiste, de l'Ordination de Grégoire, de l'Exaltation de la Sainte Croix, de Matthieu, Maurice, Denis, Bâle, Simon et Jude, et de Bénigne (une pitance).

Si nous ne pouvons tisser une équivalence parfaite entre l'échelle des rits établie plus tôt et le menu des repas, l'examen des pitances prescrites lors des jours de fêtes par les ordinaires suggère que la solennisation des fêtes liturgiques affectait cette partie de la vie temporelle des moines, les deux manuscrits attribuant aux fêtes majeures trois ou quatre pitances. Dans le passage du coutumier signalé plus tôt, l'expression: «ad scalam» fait sans doute référence à une estrade sur laquelle se tenaient dans le réfectoire l'abbé et le magister ordinis et par cela à la dignité de ces deux religieux qui leur valait un traitement de faveur au cours des repas, ce qui atteste que le système d'équivalence entre les menus et les rits était complété par un sous-régime, suivant lequel un nombre plus important de plats était présenté aux religieux de plus haute dignité, ce nombre dépendant du degré de solennité des fêtes et en particulier de l'indication du nombre de cierges allumés dans le chœur.

Les manuscrits montois témoignent d'un deuxième système d'organisation des repas, lié à l'anniversaire des défunts et complémentaire du régime de pitances dépendant du degré de solennité des fêtes: l'Ordinaire 46 (fol. 84r) et l'Ordinaire 216 (fol. 95r) signalent que le repas des moines devait être augmenté d'une pitance à l'occasion de l'anniversaire d'un comte ou d'un duc de Normandie, d'un abbé du Mont Saint-Michel et d'un archevêque de Rouen, l'Ordinaire 216 ajoutant à cette liste l'anniversaire d'un roi ou d'une reine de France. En outre, une pitance, du vin miellé et des gâteaux complétaient le repas du convent lors de l'anniversaire d'un évêque d'Avranches selon l'Ordinaire 46 (fol. 84r): cette prescription fut ramenée à une unique pitance dans l'Ordinaire 216 (fol. 95r). L'Ordinaire 46 donne, outre ces règles générales, la prescription d'une pitance, de gâteaux et de vin pour l'anniversaire d'un comte Richard ${ }^{33}$ : cette règle date sans doute du milieu du XIII ${ }^{\mathrm{e}}$ siècle, semblant correspondre à la pitance du 20 novembre fondée par l'abbé Richard Turstin en l'honneur du duc Richard $\mathrm{I}^{\mathrm{er}}$, présentée dans le manuscrit Avranches, Bibl. mun., ms 214 (p. 345) ${ }^{34}$, qui mentionne en outre fromage et poisson. Le régime de pitances pour les anniversaires était réglé par le pitancier de l'abbaye présenté dans l'obituaire-pitancier ${ }^{35}$, et dénote un aménagement des Statuts de Grégoire IX, copiés à la suite du coutumier du Mont Saint-Michel ${ }^{36}$, et de la règle de saint Benoît (chapitres 39 et 40).

33. Avranches, Bibl. mun., ms 46, fol. 84r: «Pro comite uero Ricardo pitanciam et pigmentum et gastellos...».

34. Cf. Chazelas, 1967 , p. 147.

35. Avranches, Bibl. mun., ms 215, fol. 159r-174v.

36. Avranches, Bibl. mun., ms 214, p. 11: "Ab idibus septembris usque ad capud Quadragesime, secundum regulam beati Benedicti, reficiantur ad nonam et in aliis temporibus, secundum regulam eandem, et illo moderamine ciborum quod describit regula et ordinatis pitanciis, si quando eas fieri contigerit, sint contenti, nec aliquis sibi in cibo et potu delicaciora parari uel extra deferri faciat uel recipiat presentata, set quicquid presentatum fuerit coram illo qui tunc conuentui preest deferatur et ipse taliter prouideat siue de hiis siue de aliis pitanciis, quod eorum confortet naturam qui delicati uel debiles commode communibus cibis uti non possunt». 


\section{Conclusion}

La comparaison des degrés de solennité des fêtes du sanctoral présentés dans les calendriers et les ordinaires montois éclaire l'évolution des usages liturgiques de la communauté du Mont Saint-Michel entre le XIII et le XV siècle: suivant l'attribution des rits, de vigiles et d'octaves, les fêtes de la Vierge, les fêtes de saint Michel, la fête de saint Aubert ainsi que plusieurs fêtes de saints dont l'abbaye possédait des reliques comme saint Laurent figuraient au sommet de la hiérarchie montoise des fêtes; en outre, le degré de solennité de dix-neuf fêtes fut rehaussé à la fin du Moyen Âge. On observe par ailleurs une nette connexité de la hiérarchie des fêtes et du règlement des menus prescrits par les ordinaires.

Les rits attribués aux fêtes, qui expriment des réalités cultuelles, témoignent de l'état d'une liturgie locale et ainsi de la catégorisation hiérarchique de ses fêtes à un moment de son histoire. Une application possible de l'étude des rits à l'analyse des manuscrits est d'établir, sur le fondement de ses résultats, la localisation et la datation approximative du contenu d'un calendrier liturgique. Considérons un exemple: un calendrier conservé isolément de tout livre liturgique, aujourd'hui contenu dans le manuscrit Avranches, Bibl. mun., ms 162 (fol. 8or-81v), qui servit probablement de renfort de reliure à l'époque moderne. Ce calendrier ne compte plus que quatre mois (janvier, février, novembre et décembre). La plupart des fêtes qui y sont inscrites y sont suivies d'une mention de rits; les fêtes de la Nativité, de la Circoncision du Seigneur, de l'Épiphanie, de la Toussaint, de la Conception et de la Purification de la Vierge, de la Conversion de Paul, de Catherine, d'Étienne et des Saints-Innocents, ainsi que les fêtes des apôtres André, Jean, Thomas et Mathias y sont copiées en rouge. Les saints cités par le calendrier - parmi lesquels les saints Laumer, Bénigne, Mélaine, Menne et Malo - apparaissent tous dans le sanctoral des livres du Mont Saint-Michel. La fête de saint Bénigne bénéficie en outre dans ce calendrier d'un haut degré de solennité, y recevant le rit In albis. Si elle ne peut être démontrée avec certitude à partir de ces quatre mois, l'origine montoise du calendrier apparaît ainsi au moins possible. Si l'on suit cette hypothèse, une observation des rits donnés par ce calendrier le désigne comme contemporain $\mathrm{du}$ calendrier du Collectaire 215 (XV $\mathrm{XV}^{\mathrm{e}}$ siècle) et du calendrier de l'Ordinaire 216 copié en 1432 (sans que nous puissions savoir duquel de ces deux calendriers il est le plus proche, son état fragmentaire ne permettant pas davantage de comparaisons) $)^{37}$ : ce document présente en effet le même état des rits que les manuscrits 215 et 216 d'Avranches aux fêtes de Lucie (In albis, XII lectiones), des Saints-Innocents (In capis), de Thomas Becket (In albis), de la Purification de la Vierge (In capis), de la Chaire de Pierre (In albis), de Clément (In albis) et de Catherine (In capis). Le calendrier conservé dans le manuscrit 162 se distingue en outre d'un état liturgique daté du XIV ${ }^{e}$ siècle et présenté dans l'Ordinaire 46 ,

37. La fête de sainte Barbe ( 4 décembre), qui est absente des calendriers du collectaire et du deuxième ordinaire, apparaît en outre copiée de seconde main dans le calendrier du manuscrit 162 (fol. 81v). 
dans lequel la fête de la Chaire de Pierre est fête de rit Duodecim lectiones, alors qu'elle est fête In albis dans le calendrier du manuscrit 162. Le contenu de ce calendrier peut ainsi être daté, selon une critique interne, $\mathrm{du} \mathrm{XV}^{\mathrm{e}}$ siècle.

\section{Bibliographie}

BoulC'H, Stéphane, «Le repas quotidien des moines occidentaux du haut Moyen Âge», Revue belge de philologie et d'histoire, 75-2, 1997, p. 287-328, DOI: 10.3406/ rbph.1997.4172.

CABY, Cécile, "Abstinence, jeûnes et "pitances" dans le monachisme médiéval», in Pratiques et discours alimentaires en Méditerranée de l'Antiquité à la Renaissance, Actes du colloque de la Villa Kérylos à Beaulieu-sur-Mer (4-6 octobre 2007), Jean Leclant, André Vauchez et Maurice Sartre (éd.), Paris, Académie des Inscriptions et Belles Lettres (Cahiers de la Villa Kérylos, 19), 2008, p. 271-292.

Chazelas, Jean, «La vie monastique au Mont Saint-Michel au XIII e siècle», in Millénaire monastique du Mont Saint-Michel, I: Histoire et vie monastiques, Jean LAPORTE (éd.), Paris, Lethielleux, 1967, p. 125-150.

Chevalier, Louis, Agere et statuere: étude historique et édition critique et numérique des deux ordinaires liturgiques du Mont Saint-Michel (XIV ${ }^{e}-X V^{e}$ siècles), thèse d'histoire, Université de Caen Normandie, 2019, 3 vol.

Collomb, Pascal, «Le Liber Ordinarius: un livre liturgique, une source historique», in Comprendre le XIII siècle : études offertes à Marie-Thérèse Lorcin, Pierre GUICHARD et Danièle Alexandre-Bidon (dir.), Lyon, Presses universitaires de Lyon, 1995, p. 97-109.

Dreves, Guido Maria, Analecta Hymnica Medii Aevi, 19: Hymni inediti, Leipzig, O. R. Reisland, 1895.

Duвогs, Jacques, «Le trésor des reliques de l'abbaye du Mont Saint-Michel», in Millénaire monastique du Mont Saint-Michel, I: Histoire et vie monastiques, Jean LAPORTE (éd.), Paris, Lethielleux, 1967, p. 501-593.

FiAsson, David, «Un chien couché au pied du roi d'Angleterre? Robert Jolivet, abbé du Mont Saint-Michel (1411-1444)», Annales de Normandie, 64-2, 2014, p. 47-72.

FIAsson, David, «Ravitaillement, communications et financement de la garnison du Mont Saint-Michel (1417-1450)", in La guerre en Normandie (XI ${ }^{e}-X V^{e}$ siècles), Actes du colloque international de Cerisy (30 septembre-3 octobre 2015), Anne CURRY et Véronique Gazeau (dir.), Caen, Presses universitaires de Caen (Symposia), 2018, p. 217-229.

Guilloreau, Léon, «Un fragment de coutumier de l'abbaye du Mont Saint-Michel édicté en l'an de grâce 1258", Revue catholique d'histoire, d'archéologie et littérature de Normandie, vol. 24-25, 1915, p. 161-176.

Lebigue, Jean-Baptiste, «La législation des usages liturgiques au Moyen Âge (XII ${ }^{e}$ $\left.\mathrm{XV}^{\mathrm{e}} \mathrm{s}.\right)$ ), Revue de l'histoire des religions, 229-3, 2012, p. 349-373.

LEBIGUE, Jean-Baptiste, «Rits et couleurs: acronymie et chromonomie des calendriers liturgiques au Moyen Âge », in Le manuscrit enluminé: études réunies en hommage à Patricia Stirnemann, Claudia Rabel (éd.), Paris, Le Léopard d'or (Cahiers du Léopard d'or, 16), 2014, p. 39-73. 
LemariÉ, Joseph, «La vie liturgique au Mont Saint-Michel d'après les ordinaires et le cérémonial de l'abbaye», in Millénaire monastique du Mont Saint-Michel, I: Histoire et vie monastiques, Jean LAPORTE (éd.), 1967, p. 303-352.

Martimort, Aimé-Georges, Les ordines, les ordinaires et les cérémoniaux, Turnhout, Brepols (Typologie des sources du Moyen Âge occidental, 56), 1991.

Neufville, Jean (éd.), La Règle de saint Benoît, Adalbert de VogüÉ (introd. et trad.), Paris, Cerf (Sources chrétiennes), 1972-1977, 7 vol.

The Ordinal of the Abbey of the Holy Trinity Fécamp (Fécamp, Musée de la Bénédictine, Ms. 186), vol. 2, David ChadD (éd.), Woodbridge, Boydell Press (Henry Bradshaw Society, 112), 1999-2002.

TARDIF, Henri, «La liturgie de la messe au Mont Saint-Michel aux XI ${ }^{\mathrm{e}}$, XII ${ }^{\mathrm{e}}$ et XIII ${ }^{\mathrm{e}}$ siècles", in Millénaire monastique du Mont Saint-Michel, I: Histoire et vie monastiques, Jean LAPORTE (éd.), 1967, p. 353- 377.

«Traité pour la défense du Mont Saint-Michel en 1440 », Mémoire de la Société des antiquaires de Normandie, XV, 1846, p. 212-213.

Trotin, Nicolas, «La Trinité de sainte Anne, un avatar du dogme immaculiste: à propos d'une planche gravée par Simon Vostre (1508)», in Marie et la "fête aux Normands »: dévotion, images, poésie, Actes du colloque de Rouen (janvier 2009), Françoise Thelamon (dir.), Mont-Saint-Aignan, Presses universitaires de Rouen et du Havre, 2011, p. 153-163.

VogüÉ, Adalbert de, «Travail et alimentation dans les Règles de saint Benoît et du Maître», Revue bénédictine, 74, 1964, p. 242-251.

\section{Catégorisation hiérarchique des fêtes}

du sanctoral des ordinaires du Mont Saint-Michel

(Avranches, Bibl. mun., ms 46 et Avranches, Bibl. mun., ms 216)

- Fêtes in capis et à douze leçons: Aubert; Jean-Baptiste; Apparition de Michel sur le Mont Gargan; Jean; Annonciation; Conception de la Vierge; Purification de la Vierge; Assomption; Nativité de la Vierge (fêtes in capis, à douze leçons et trente cierges). Michel - 29 septembre (fête à douze leçons et trente cierges). Laurent (fête in capis, à douze leçons et neuf cierges). Benoît; Translation de Benoît; André; Nicolas; Marie-Madeleine; Saints-Innocents; Pierre et Paul; Étienne; Martin; Catherine (fêtes in capis, à douze leçons et sept cierges).

- Fêtes in albis, à douze leçons et cinq cierges: Lucie; Thomas Becket; Sébastien; Agnès; Vincent; Conversion de Paul; Agathe; Mathias; Grégoire; Ambroise; Marc; Philippe et Jacques apôtres; Inventio de la Sainte-Croix; Jean l'évangéliste; Translation de Nicolas; Barnabé; Paul; Martin; Jacques apôtre; Pierre; Inventio d'Étienne; Thomas apôtre; Exaltation de la SainteCroix; Wandrille; octave de l'Assomption; Louis; Ouen; Barthélemy; Augustin; Décollation de Jean-Baptiste; Ordination de Grégoire; octave de la Nativité de Marie; Matthieu; Maurice; Côme et Damien; octave de Michel; Denis; Luc; Simon et Jude; Bénigne; Cécile; Clément. 
- Fêtes à douze leçons et trois cierges: octave de Jean; Maur; Julien; Blaise; Scholastique; Georges; Marcellin; Gervais et Protais; octave d'Aubert; octave de Jean-Baptiste; octave de Pierre et Paul; Germain; Chaire de Pierre; Paterne; Nicaise; Melaine; Brice; Malo; octave de Martin; Colomban.

- Fêtes à trois leçons: octave d'Étienne; octave des Saints-Innocents; Marcellus; Prisca; Laumer; Émérance; Polycarpe; octave d'Agnès; Brigitte; Dorothée; Valentin; Albin; Tiburce et Valérien; Vital; Athanase; Gordien et Épimaque; Nérée, Achillée et Pancrace; Basile; Urbain; Chéron; Nicomède; Marcellin et Pierre; Médard; Prime et Félicien; Basilide, Cyrin et Nabor; Guy, Modeste et Crescence; Cyr et Julitte; Leufroy; vigile de Jean-Baptiste; Jean et Paul; Léon; Processus et Martinien; les Sept Frères; Marguerite; Praxède; Anne; les Sept Dormants; Félix; Abdon et Sennen; Étienne; Felicissime et Agapit; Donat; Large et Smaragde; Cyriaque; Romain; Hippolyte; Eusèbe; Rufus; Félix et Audacte; Lucie et Géminien; Lambert; Gérard; Calixte; Bâle; Crépin et Crépinien; Quentin; les Quatre Couronnés; Théodore; Saturnin; Chrysogone.

- Memoria: Damase; Fabien; Alexandre, Evence et Théodore; Marc et Marcellin; Martial; Berthevin; Arnoul de Metz; Christophe et Cucufat; Arnoul de Soissons; Timothée et Symphorien; Julien de Brioude; Adrien; Euphémie; Gorgon; Prote et Hyacinthe; Piat; Léger; Léonard; Menne. 